\title{
Effect of Fuel Sootiness on the Heat Fluxes to the Walls in Enclosure Fires
}

\author{
P. TOFILO, M.A. DELICHATSIOS, and G.W.H. SILCOCK \\ Institute for Fire Safety Engineering Research \& Technology (FireSERT) \\ University of Ulster \\ Jordanstown, BT37 0QG, UK
}

\begin{abstract}
Even though considerable work has been performed regarding gas temperatures and burning rates in enclosures, scant information is available for the heat fluxes and their distributions on the walls of an enclosure. These heat fluxes are necessary input for determining the thermal response and performance of the wall materials and especially glazing. The heat fluxes on the wall of an experimental enclosure were deduced from the temperatures in several thermally thin small steel plates $(25.4 \mathrm{~mm} \times 25.4 \mathrm{~mm} \times 3 \mathrm{~mm}$ thick) and in the insulation surrounding the steel plates. In addition, the mass loss rate and gas temperatures near these heat flux gauges were measured. The experimental enclosure was the $1 / 3$ linear scale of the ISO room corner test having six openings and three square- pans of variable size burning Methanol, IMS (Industrial Methylated Spirits) and Toluene at the corner and in the center of the enclosure. By choosing fuels with an increased degree of sootiness it was found that the heat fluxes do not depend only on the gas temperature, as claimed before for heat fluxes on the floor, but also on the magnitude of the heat release rate and the fuel sootiness. Moreover, comparison of the heat fluxes for methanol, IMS and toluene allow the separation of convective from radiative heat fluxes owing to hot gases (and the enclosure walls) and from the radiative heat fluxes from the fire plume.
\end{abstract}

KEYWORDS: heat fluxes, enclosures, sooty fuels, radiation

\section{NOMENCLATURE LISTING}

$\begin{array}{clcl}T_{0} & \text { ambient temperature } & c & \text { specific heat of carbon steel } \\ T_{g} & \text { gas temperature } & & \\ T_{s} & \text { measured steel temperature } & A_{0} & \text { area of the opening } \\ T_{w} & \text { wall temperature } & H_{0} & \text { height of the opening } \\ \dot{q}_{\text {rad }}^{\prime \prime} \text { fames } & \text { radiative heat flux from the flames } & A_{F} & \text { area of the fuel bed } \\ \dot{q}_{\text {rad hotalyer }}^{\prime \prime} & \text { radiative heat flux from hot layer } & & \\ \dot{q}_{W}^{\prime \prime} & \text { radiative heat flux from hot walls } & \text { Greek } & \\ \dot{q}_{c}^{\prime \prime} & \text { convective heat flux } & \sigma & \text { Steffan-Boltzmann constant } \\ \dot{q}_{\text {cond }}^{\prime \prime} & \text { conduction losses } & \rho & \text { Density of carbon steel } \\ h_{c} & \text { heat transfer coeeficient } & \delta & \text { Thickness of the steel plate }\end{array}$

\section{INTRODUCTION}

Prediction of heat fluxes in enclosure fires is an essential and necessary input in determining (a) fire spread and fire growth and (b) the response of non-combustible elements (e.g., steel, concrete, glazing) to the fire. The magnitude of the heat fluxes depends on the sootiness of the burning materials, the size of the fire, the geometry of the enclosure and the burning conditions (well or under ventilated) inside an enclosure.

FIRE SAFETY SCIENCE-PROCEEDINGS OF THE EIGHTH INTERNATIONAL SYMPOSIUM, pp. 987-998

COPYRIGHT @ 2005 INTERNATIONAL ASSOCIATION FOR FIRE SAFETY SCIENCE 
Considerable work has been done in this area [1,2] but there is not a definitive and systematic way to determining the heat fluxes either from similarity correlations or from CFD (computational fluid dynamics) calculations. This work fills some of the gaps by investigating the heat fluxes on the walls of an enclosure from square pan fires burning liquid fuels with increased degree of sootiness i.e., methanol, IMS (Industrial Methylated Spirits) and toluene. One goal is to separate the three main components of this heat flux i.e., radiation from the hot layer (and the enclosure walls), radiation from the flames and convection from the gases around the point of interest. The last component is very important for the estimation of the breakage of glazing because the glazing wholly absorbs convective fluxes, in contrast to radiation fluxes.

The present paper presents results for pan fires in an enclosure of linear size equal to $1 / 3$ of the ISO room corner and openings of different sizes. The structure of the paper is the following. The experimental setup and procedure are outlined first including the design of a new heat flux gauge, which consists of thermally thin steel plates surrounded by insulation except for the exposed surface. The heat flux is deduced from the temperature in the steel plate and the insulation. A comprehensive prediction methodology is pursued from the experimental data by correlating (a) the mass pyrolysis rates with the opening factor (also called ventilation factor $A_{0} \sqrt{H_{o}}$ ) and the size of the pan [3,4] and (b) the heat flux with the local gas temperature. There are ongoing efforts to produce a correlation for determining the gas temperatures in terms of the heat release rates, the enclosure geometry and the size of the opening. Preliminary results of this correlation are included in a previous publication [5].

\section{EXPERIMENTAL SETUP AND PROCEDURE}

Mass loss rates of square pan fires, wall heat fluxes and gas temperatures were measured in an enclosure 1/3 the ISO Room size ( $0.8 \mathrm{~m} \times 1.2 \mathrm{~m}$ x $0.8 \mathrm{~m}$ high $)$ as shown in Fig. 1. The walls and ceiling had an inner layer $25 \mathrm{~mm}$ thick insulation board (Fiberfax) and outer layer $10 \mathrm{~mm}$ thick fire retarded chipboard. The floor was made of high density fibreboard. The enclosure has an opening of variable size as shown in Fig. 2.

The fuels were Methanol (purity 99\%), Industrial Methylated Spirits IMS (composition: ethanol $91 \%$, methanol $4 \%$, impurities and water 5\%) and Toluene burned in square pans of size $0.2 \times 0.2 \mathrm{~m}, 0.25 \times 0.25 \mathrm{~m}$ and $0.3 \times 0.3 \mathrm{~m}$. Each pan was a) filled up to the same height with an amount of fuel as listed in Fig. 2 resulting in a quasi-steady period of burning and b) located at the center or corner of the enclosure for the IMS experiments and only at the corner for the Methanol and Toluene experiments. Total heat fluxes were measured at 25 points on one wall (see Fig. 1) by using thermally thin steel plates as explained later in this paragraph. In addition, five Gardon heat flux gauges placed next to the center column of the steel plates measured the heat fluxes for comparison and validation of the steel plate measurements. Gas temperatures were measured through a thermocouple tree having nine thermocouples near the instrumented wall from which five were next to the center column of steel plates as illustrated in Fig. 1. Data was collected after ignition of the fuel at every four seconds until all fuel was consumed. The new heat flux gauge (having similarities with the plate thermometer [6]) allows mapping of heat fluxes at several positions of the wall area at a cost considerably smaller than the cost if Gardon Gauges were used. The steel plate has dimensions $25.4 \mathrm{~mm}$ x $25.4 \mathrm{~mm}$ x $3 \mathrm{~mm}$ thick and is surrounded by Fibrefax insulation $25 \mathrm{~mm}$ thick except for the exposed surface as shown in Figs. 3 and 4a. 


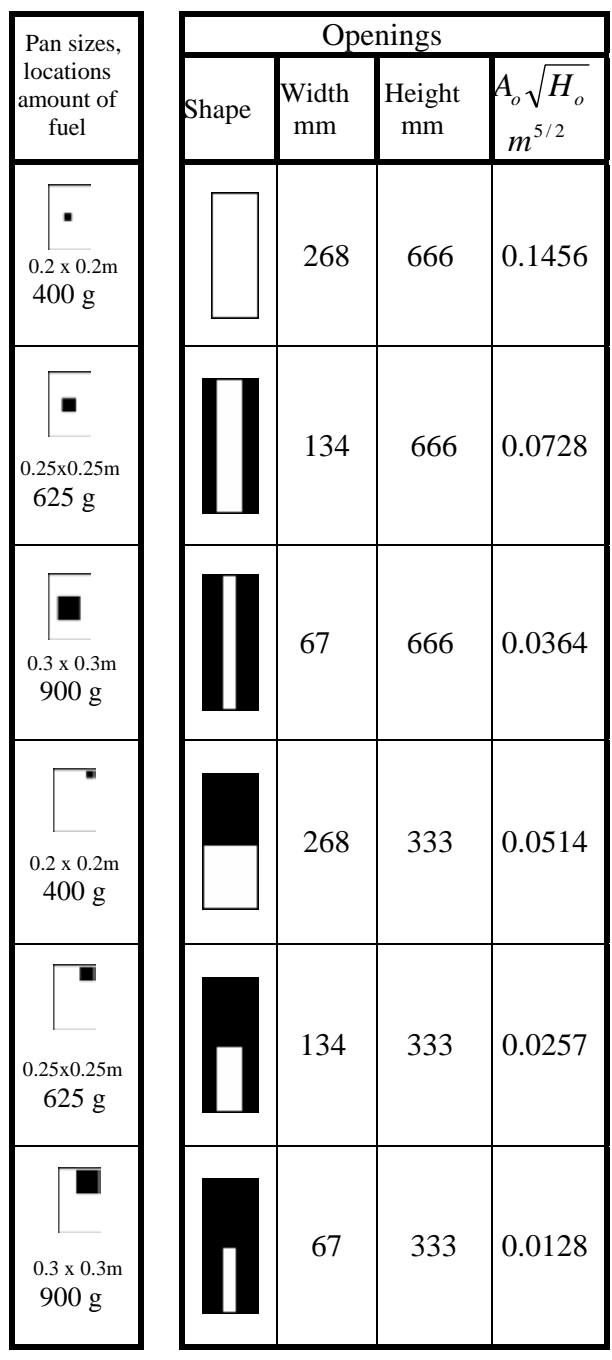

Fig. 2. Openings and pan sizes.

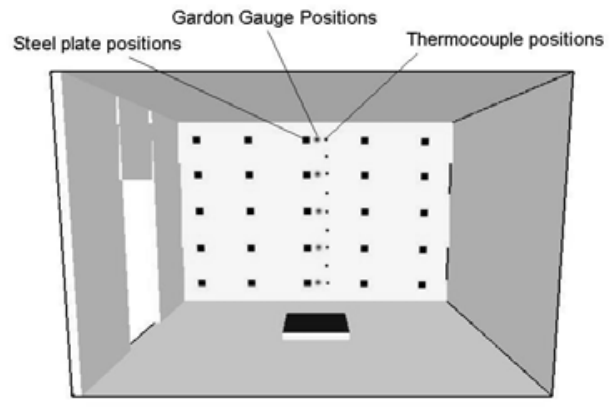

Fig. 1. Enclosure and measurement points.

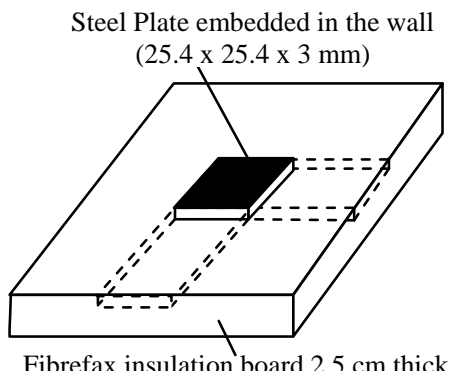

Fig. 3. Steel plate surrounded by insulation.

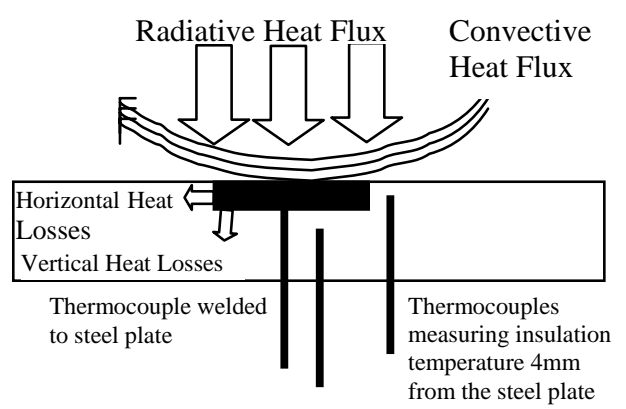

Fig. 4a. Design and concept of measurement.

For the deduction of the heat fluxes, the temperature is measured at the center of the unexposed side of steel plate, which behaves as thermally thin, and at two positions in the surrounding insulation as shown in Fig. 4a. The front surface of the plate is covered with carbon black to ensure that its emissivity is one. This design has the advantage over a design where a large plate of steel [7] is used that the lateral conduction losses are reduced, so that the heat flux measurement can be more reliable over the whole heating history even if there are large spatial variations of heat fluxes. The heat flux is deduced by accounting for the heat stored in the thermally thin plate, the conduction heat transferred between steel plate and surrounding insulation, a radiation correction term and a convection correction term. The correction terms allow the determination of the heat flux for a reference temperature equal to the initial temperature of the surface i.e., 
rendering this value equivalent to the heat flux measured by the Gardon gauge. In conclusion, the heat flux deduced from the steel plate is:

$\dot{q}^{\prime \prime}=\rho c \delta \frac{d T_{s}}{d t}+\dot{q}_{c o n d}^{\prime \prime}+h_{c}\left(T_{s}-T_{o}\right)+\sigma\left(T_{s}^{4}-T_{o}{ }^{4}\right)$

All the quantities in this equation are directly measured or calculated from measured quantities. The plate is thermally thin of thickness $\delta(3 \mathrm{~mm})$, density $\rho\left(7850 \mathrm{~kg} / \mathrm{m}^{3}\right)$, and specific heat c (temperature dependant $\sim 500 \mathrm{~J} / \mathrm{kg} \mathrm{K}$ ) whereas the insulation conductivity is $\sim 0.09 \mathrm{~W} / \mathrm{mK}$. By comparison with the Gardon gauge measurements and the experimental results for Methanol and IMS, it was found, that (a) the convective heat transfer coefficient is constant equal to $15 \mathrm{~W} / \mathrm{m}^{2} \mathrm{~K}$ (Fig.15) and (b) the conduction losses are half the value calculated from the temperature measurements on the plate and the two locations in the insulation using simple minded heat transfer calculations from the sidewall surface and the bottom surface of the steel plate. This procedure was validated by comparison of all the steel plate measurements at the center of the instrumented wall (i.e., Eq. 1) with the corresponding Gardon gauge heat flux measurements as shown for one case in Fig. $4 \mathrm{~b}$.

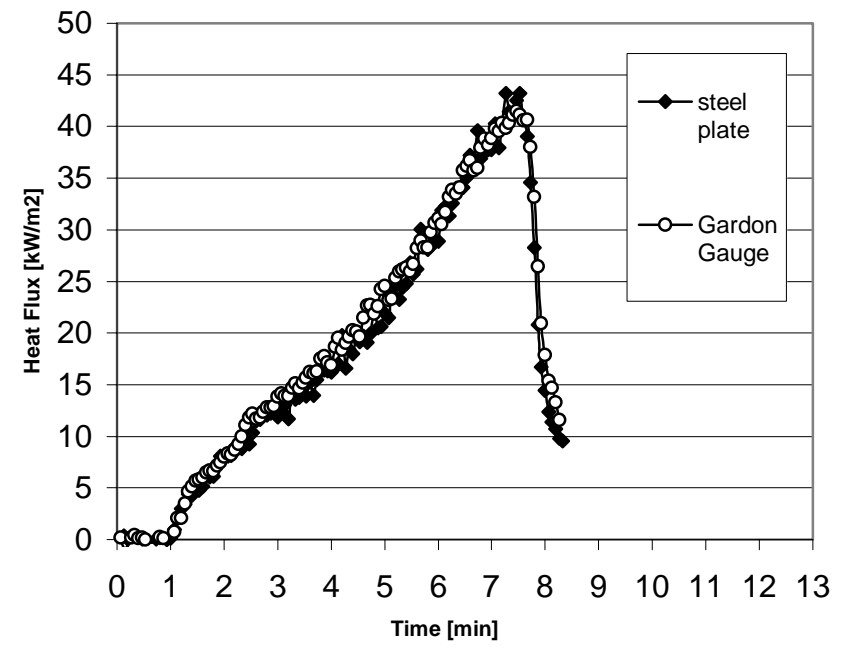

Fig. 4b. Validation of the steel plate method (one case - Exp. 7, topmost location).

\section{RESULTS}

Figure 5 depicts and numbers for quick reference the 72 experimental conditions of this work with three different fuels. Selected duplicate experiments reproduced nearly the same results. All data is available electronically. As an example, data for the IMS fuel and the medium pan size fires $(0.25 \mathrm{~m} \mathrm{x} 0.25 \mathrm{~m})$ is shown in Figs. $6(\mathrm{a}, \mathrm{b}, \mathrm{c})$ and $7(\mathrm{a}, \mathrm{b}, \mathrm{c})$ for the center and the corner fire locations, respectively, and for all opening sizes. Each figure includes the histories for the mass loss, the maximum gas temperature and the 


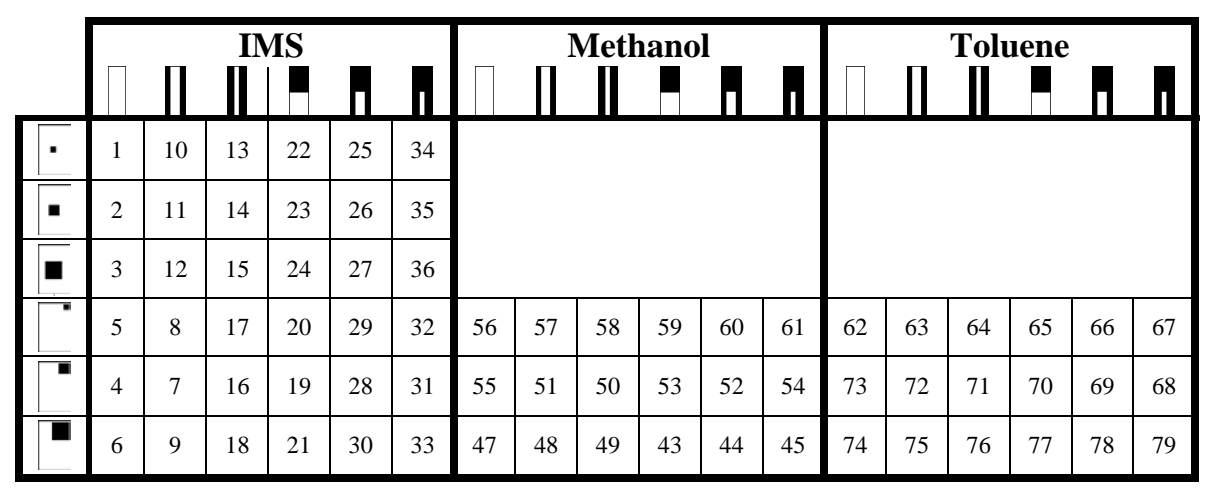

Fig. 5. Matrix and numbering of experiments.
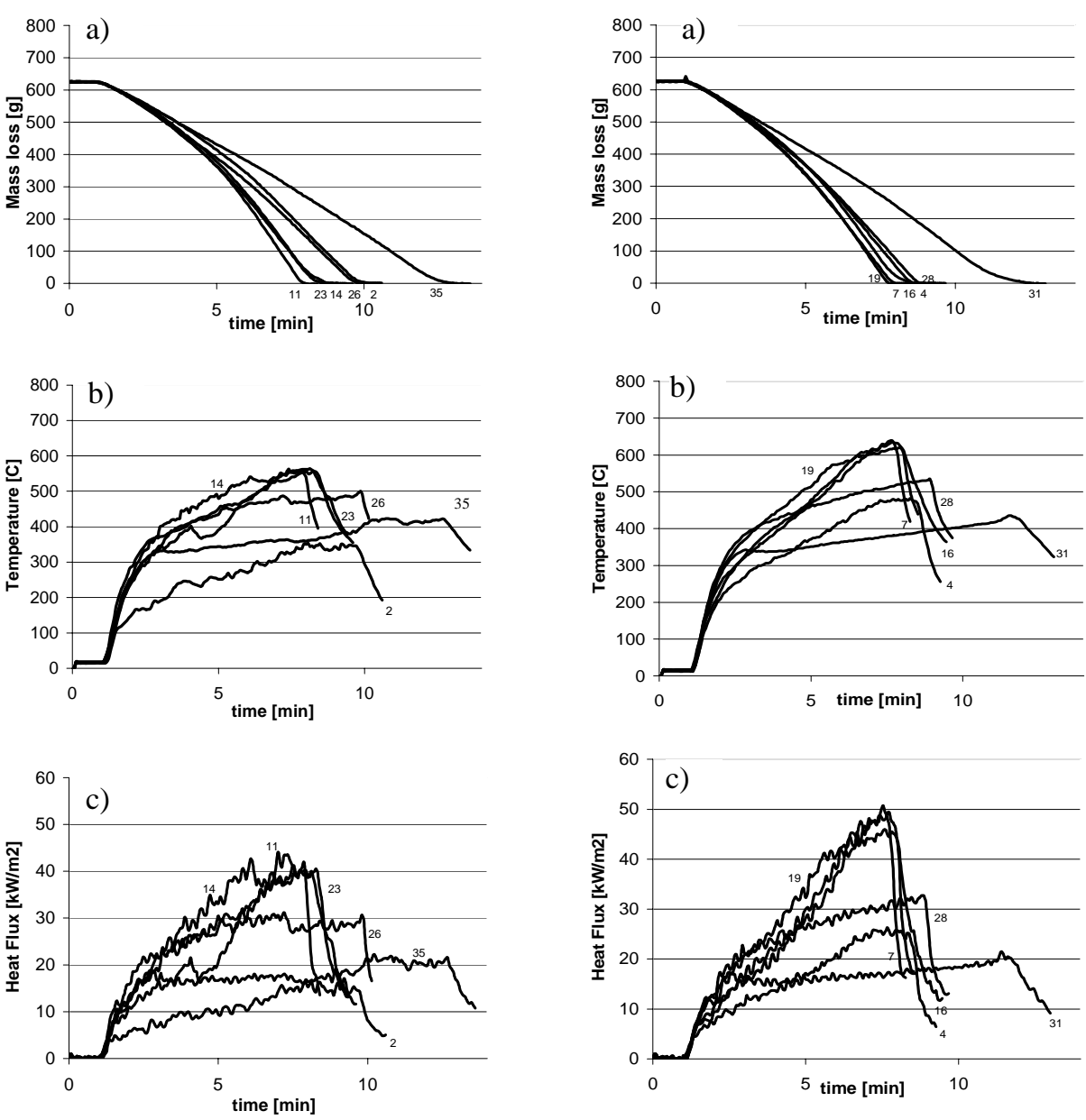

Fig. 6a,b,c. Mass loss, temperature and heat flux for the IMS centre fires.

Fig. 7a,b,c. Mass loss, temperature and heat flux for the IMS corner fires. 
(maximum) heat flux measured at the topmost centerline measurement point. Two important observations from these figures are:

1. As expected, gas temperatures for fires at the corner are larger than the ones for fires at the center because less air is entrained in fires at corners.

2. A significant increase of the fire intensity relative to the largest ventilation factor i.e., experiment 2 is noticeable for the openings with the second and third highest ventilation factor i.e., experiments 11 and 23 for center and 7 and 19 for corner fires (see Fig. 2 and Fig. 5.). Larger temperatures, heat fluxes and mass loss rates manifest this increase in Fig. 6 and Fig. 7.

Visual observations and video recordings show that the luminosity of the flames decreased as the size of the opening decreased, presumably mirroring a change of the flames from non-premixed to partially premixed. The decrease in luminosity is probably due to a decrease of the radiation from the flames with concomitant decrease of mass loss rates and heat fluxes.

\section{ANALYSIS OF THE EXPERIMENTAL RESULTS}

A methodology for predicting heat fluxes in any enclosure and fire size is pursued from the present experimental data by (a) correlating the mass pyrolysis rates with the opening factor and the size of the pan (b) correlating the heat flux with local gas temperature. This methodology will be completed in another paper by determining the gas temperatures in terms of the heat release rate, the enclosure geometry and the size of the openings. Preliminary results of the last correlation are included in a previous publication [5].

\section{Mass Loss Rates for Different Sizes of Pan Fires, Openings and Fuels}

The mass loss rate derives from differentiation of the mass loss histories (see Figs. 6a and 7a). It varies with time but for the analysis the maximum nearly quasi-steady mass loss rate is used that occurs about four to six minutes after ignition (see Figs. 6a and 7a). According to previous work [3,4], the mass loss rate is normalized by the surface area of the fuel $\left(\dot{\mathrm{m}}_{\max } / \mathrm{A}_{\mathrm{F}}\right)$ and plotted in Fig. 8a for IMS and in Fig. 8b for IMS, Metahnol and Toluene against the opening factor also normalized by the fuel area $\left(A_{0} \sqrt{H_{0}} / A_{F}\right)$.

Notice that the maximum of the burning rate increases and is expected to occur at higher values of the ventilation factor for the toluene because toluene has greater stochiometric ratio $(\sim 15)$ than oxygenated fuels $(\sim 6)$ (see e.g., $[3,4])$. In difference with the results in [3,4], the data in Fig. 8a seem to be grouped according to the pan sizes (shown in the legend of Fig. 8a) although they appear to follow the same trend. Namely, for the large pan fire $(0.3 \mathrm{~m})$ the slope of the normalized mass loss rate with respect to the normalized ventilation factor is about 0.1 as expected [3,4]. However this slope decreases as the pan size decreases. In addition, the normalized maximum mass loss rate also decreases as the pan fire decreases.

A possible explanation for these behaviors of the mass loss rate in Fig. 8a is inferred by inspecting the gas temperatures histories inside the enclosure for the three pan sizes at the different openings as sampled in Fig. 8c. It is seen there that the temperatures become lower for all the openings (large and small) as the size of the pan reduces owing to the increased significance of heat losses to the walls for smaller fires. Only the data for the 
large pan fire create temperatures high enough for the analysis and correlations in $[3,4]$ to be applicable.

Finally, for large values of the normalized ventilation factor the mass loss rate per unit area tends to its free burning value.
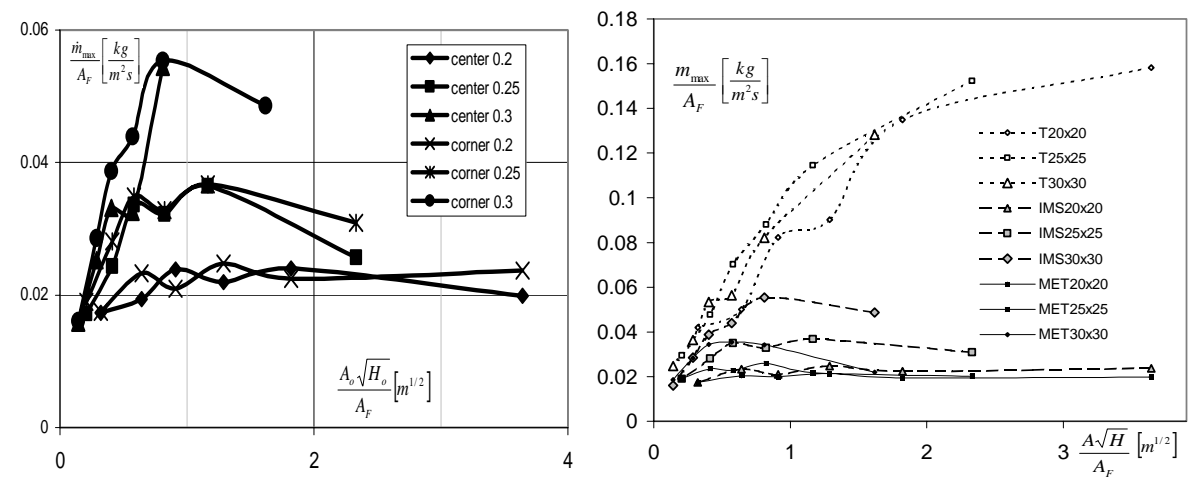

Fig. 8a and 8b. Normalized mass loss rate per fuel area against normalized ventilation factor per fuel area for a) IMS corner/centre

b) IMS, methanol and toluene corner fires.
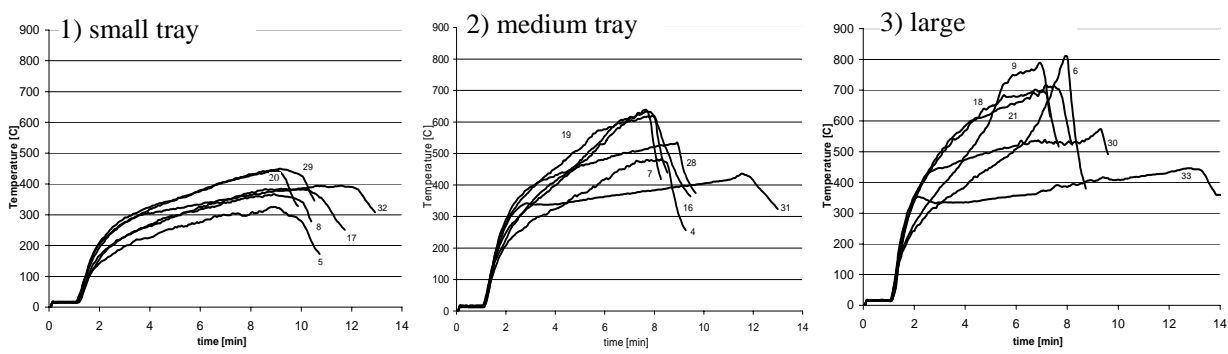

Fig. 8c (1,2,3). Gas temperatures for three pan sizes (IMS corner fires).

\section{Wall Heat Fluxes Dependance on Gas temperatures}

The heat flux on any point at the wall consists of: (a) radiation from the hot layer (and the enclosure walls) (b) convection from the gases at the point of interest and (c) radiation from the flames. With reference to a surface at the ambient temperature the heat flux is calculated from Eq.1 and can be represented as:

$$
\dot{q}^{\prime \prime}=\left(\dot{q}_{\text {rad, hotlayer }}^{\prime \prime}+\dot{q}_{w}^{\prime \prime}\right)+\dot{q}_{c}^{\prime \prime}+\dot{q}_{r a d, \text { flames }}^{\prime \prime}
$$

The first two terms on the right hand side of this equation depend on the gas temperature and the temperature of the enclosure walls. These radiation terms are expressed as:

$$
\begin{aligned}
& \dot{q}_{\text {rad,hotlayer }}^{\prime \prime}=\varepsilon_{g} \sigma\left(T_{g}^{4}-T_{o}^{4}\right) \\
& \dot{q}_{w}^{\prime \prime}=\left(1-\varepsilon_{g}\right) \sigma\left(T_{w}^{4}-T_{o}^{4}\right)
\end{aligned}
$$


assuming a view factor one for the radiation received by the heat flux plates from the walls and denoting the gas emissivity by $\varepsilon_{g}$. The convective heat flux is:

$\dot{q}_{c}^{\prime \prime}=h_{c}\left(T_{g}-T_{o}\right)$

If the heat transfer to the wall is high enough and the conductivity of the wall is low, the wall temperature is equal to the gas temperature so that the term in parenthesis in Eq. 2a becomes independent of the gas emissivity:

$\left(\dot{q}_{\text {rad,hotlayer }}^{\prime \prime}+\dot{q}_{w}^{\prime \prime}\right)=\sigma\left(T_{g}{ }^{4}-T_{o}{ }^{4}\right)$

The last term in Eq. 2a is the radiation from the flames, which depends on the radiant output of the flames, the distance from the flames and the absorptivity of the gases between the flames and the point of interest. The magnitude of this term might be evaluated by comparing the heat fluxes at two points having different distances from the fire or by comparing the heat fluxes at the same point from different size fires. In conclusion, Eqs. 2a-2e lead to the following expression for the heat flux:

$\dot{q}^{\prime \prime}=\sigma\left(T_{g}^{4}-T_{o}^{4}\right)+h_{c}\left(T_{g}-T_{o}\right)+\dot{q}_{\text {rad, flames }}^{\prime \prime}$

The analysis in Eq. 2a-2f is interrogated in Figs. 9 (a,b,c) and 10 (a,b,c) which plot the heat fluxes for the IMS fuel at the top center heat flux gauge (see Fig. 1) for locations of the fire at the center and in the corner of the enclosure, respectively. In these plots, 9a and 10a refer to small, $9 \mathrm{~b}$ and $10 \mathrm{~b}$ refer to medium and 9c and 10c refer to large pan sizes where the heat flux at a given time is plotted against the gas temperature at the same time. In addition, the curve insert depicts the radiation flux assuming that the gas radiates as a black body i.e., $\sigma\left(T_{g}{ }^{4}-T_{o}{ }^{4}\right)$. (note that heat flux measurements, not included here, at the center heat flux gauges show similar trends as those in Fig. 9 and 10)

Inspection of Figs. 9a, 10a for the small fires and Figs. 9b, 10b for the medium fires shows that (a) the heat flux depends only on the gas temperature regardless of the location or heat release rate of the fire and (b) its magnitude is higher than the flux received assuming that the gas radiates as a black body. These results together with Eq. 2f signify first that the radiative heat flux from the flames is negligible and second that the difference between the measured heat flux and that due to the blackbody radiation is the convective heat flux to the wall.

However, the heat flux data in Figs. 9c and 10c for the large fires make clear that the radiative heat flux from the flames becomes significant as the heat release rate from the fire increases. This point is further demonstrated by separating the data for the corner fires of Fig. 10c into two plots: (a) one with the smaller openings, Fig. 11a and (b) one with the larger openings Fig. 11b. As the opening size decrases (see Fig. 5 for identification of test conditions) the heat fluxes decrease because the flames and their radiative heat fluxes become weaker. The observations, analysis and conclusions in this section are strengthened by comparing the heat flux results for IMS with those for methanol and toluene in the next section. 
a)

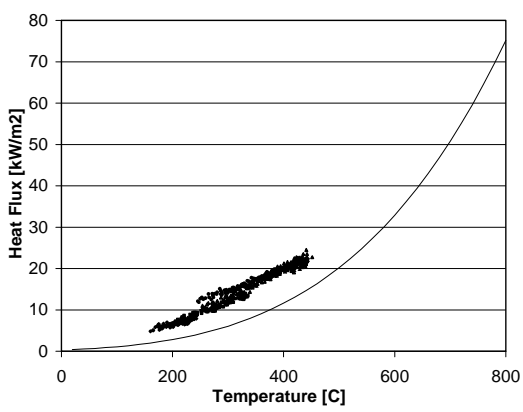

b)

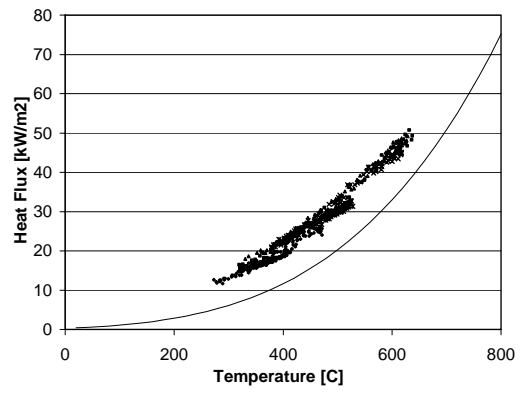

c)

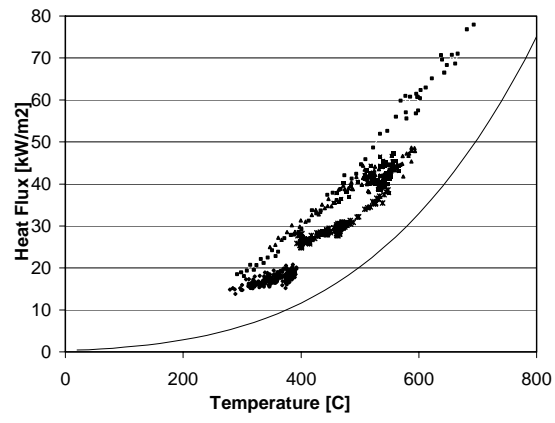

Figs. 9 a,b,c. Heat flux versus gas temperature for centre fires.

a)

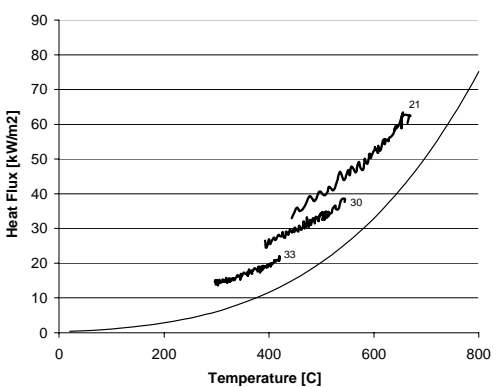

a)

b)

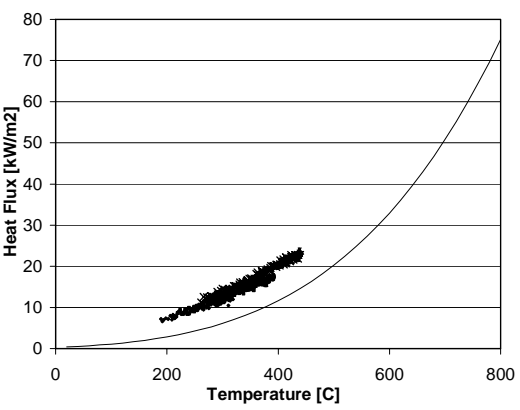

c)
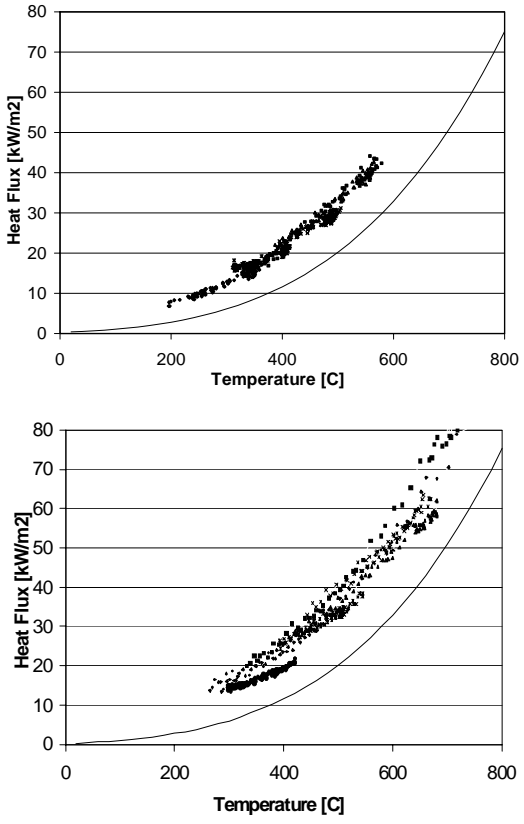

Figs.10 a,b,c. Heat flux versus gas temperature for corner fires.

b)

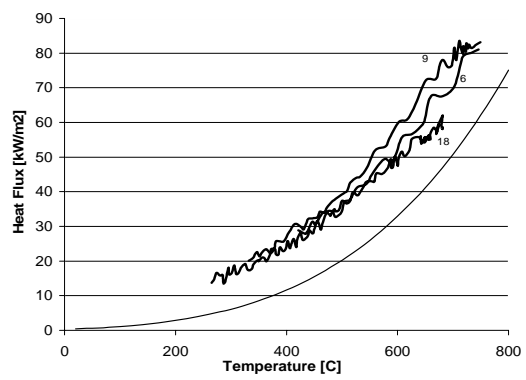

Figs. 11a,b. Heat flux at the top center of instrumented wall against gas temperature for the small (a) and large openings (b) having the large pan at the corner (solid line - black body radiation). 


\section{Effects of Fuel Sootiness}

The behaviour of methanol (non luminous) and toluene (very sooty) is compared with the behaviour of IMS (intermediate sootiness) by plotting the temperature histories and the correlation of the wall heat flux with the gas temperature. The gas temperatures histories for the three fuels are plotted in Figs. 12a and 12b for the medium pan fire at the corner having a large (tests 7,51,72) and a small opening, respectively (tests 32,61,67). Moreover, Figs. 13a and 13b plot the heat flux correlations for the same fires. The comparison and conclusions about their fire behaviour become more transparent by plotting also the heat flux correlations for IMS, Methanol and Toluene in Figs. 14a to $14 \mathrm{c}$, which include data from the experiments with the medium pan fire located at the corner of the enclosure.

Inspection of these Figures leads to the following significant remarks:

1. Fig. 12a shows that for over ventilated fires (large openings) more sooty fuels reach maximum temperatures faster than less sooty ones whereas Fig. 12b shows that for under-ventilated fires (small openings) all fuels produce almost the same temperature histories. This happens because for over ventilated fires flame radiation from sooty fuels leads to a fast increase of wall temperatures and hence, enhanced heat feedback to the fuel surface that induces larger burning rates and temperatures. On the other hand, for under-ventilated fires it seems that flame radiation does not depend on the sootiness of the fuel because presumably the flames are partially premixed and the heat release rate is small.

2. Figs. 13a and $13 \mathrm{~b}$ for the heat flux measurements corroborate the previous observations regarding the importance of the flame radiation. In addition, they show that the heat fluxes for the Methanol and IMS medium size fires are the same and hence, are not affected by the flame radiation.

3. Consistently with the previous remarks, Figs. 14a (methanol, medium fire, all openings) and 14b (IMS, medium fire, all openings) imply that the heat flux is independent of the heat release rate and the flame radiation or fuel sootiness whereas Fig. 14c (Toluene, medium fire, all openings) shows that the effects of sootiness and heat release rates are increasingly significant for larger openings. Remarkably, when the Toluene fires reach their maximum values the heat fluxes take the same values as for the less sooty fuels because the optically thick gases absorb their flame radiation.

4. The previous remarks in 1-3 indicate that the difference between the measured heat flux and the theoretical blackbody gas radiation for IMS and methanol is the convective heat flux as explained in the previous section from Eqs. 2a-2e. Fig. 15 depicts an estimate of the variation of the convective heat transfer coefficient with time derived from this heat flux by dividing it with the difference between the gas and initial temperature. The magnitude of the coefficient calculated this way remains constant for the burning duration of the experiment with values about $15 \mathrm{~W} / \mathrm{m}^{2} \mathrm{~K}$ for all fires apart from the most under-ventilated case where the value is about $12 \mathrm{~W} / \mathrm{m}^{2} \mathrm{~K}$. Comparison with ISO room scale experiments [8,9] show that the heat transfer coefficient does not depend on scale. 

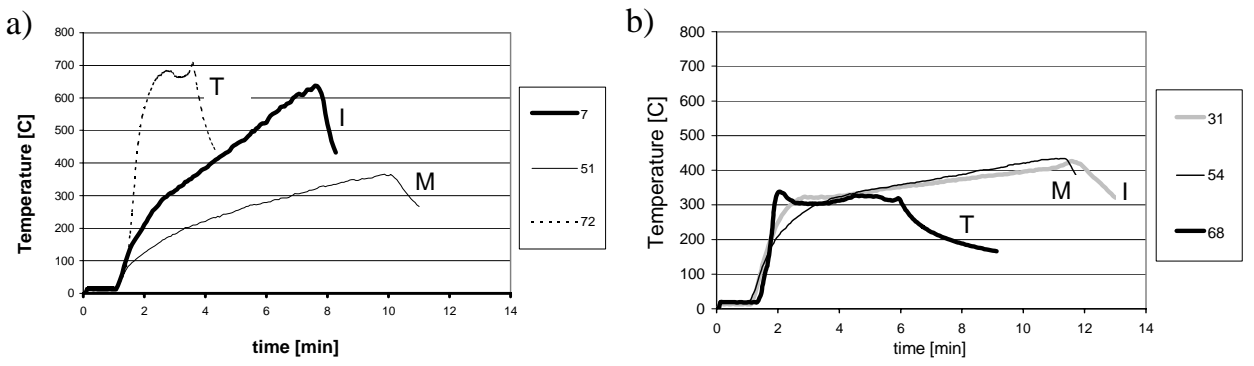

Figs. 12a,b. Gas temperatures for three fuels a) large opening b) small opening.
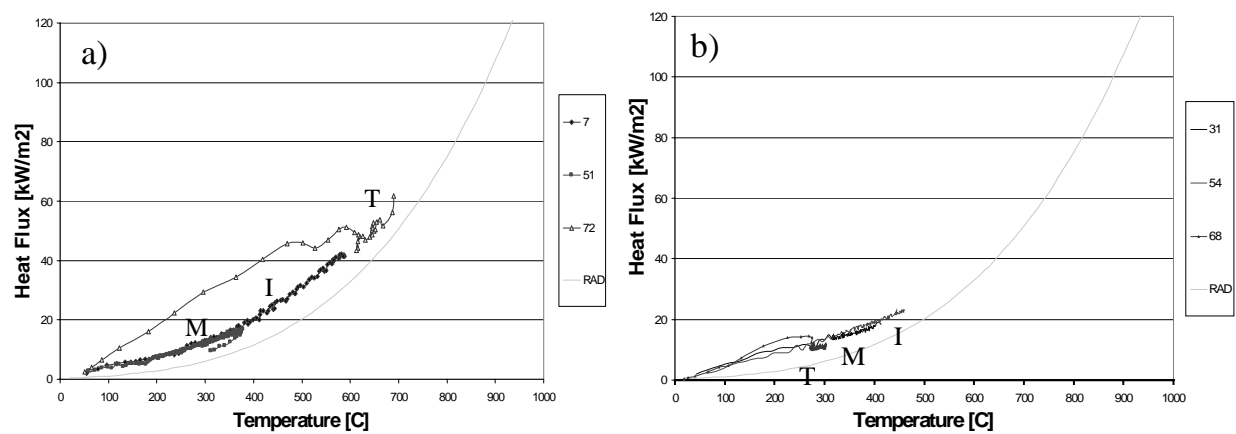

Figs. 13a,b. Heat flux vs. temperature for a) large opening b) small opening.
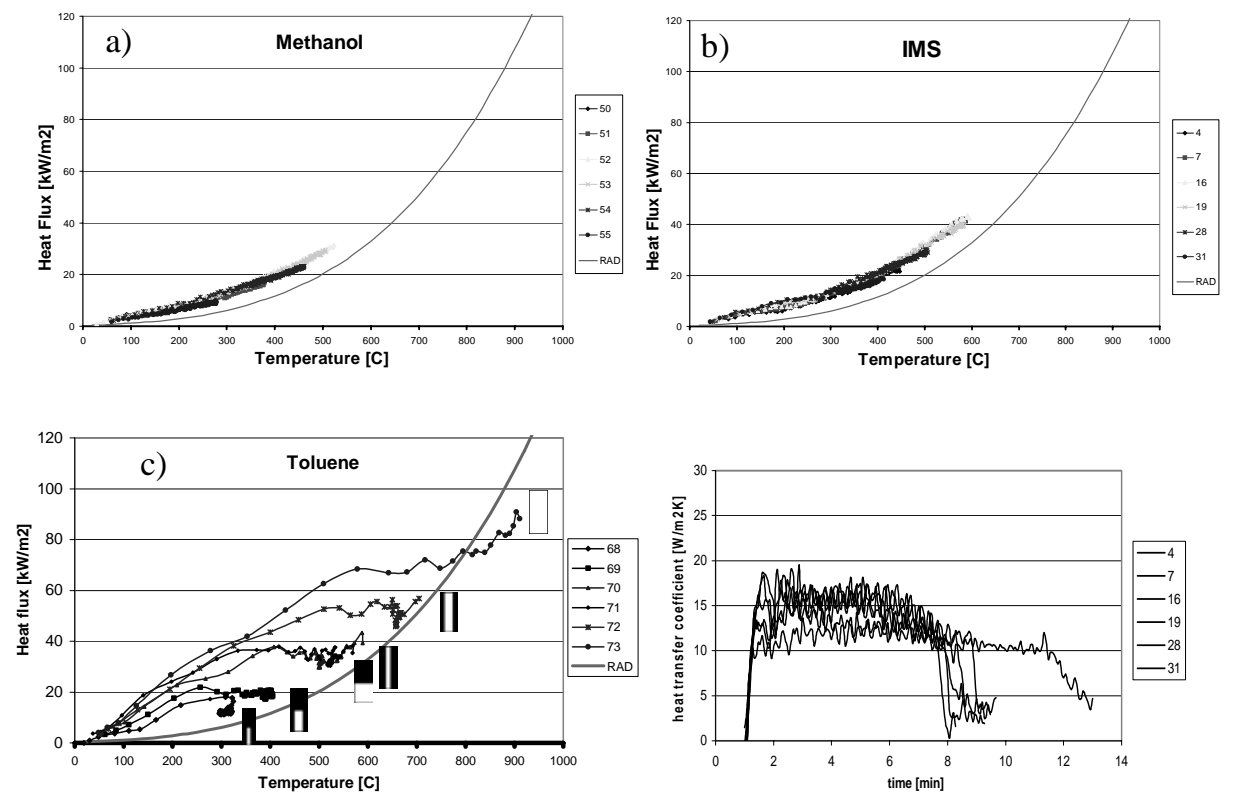

Fig. 14a,b,c. Heat flux vs temp for all fuels.

Fig. 15. Heat transfer coefficient (IMS corner). 


\section{CONCLUSIONS}

This work being part of continuing effort at FireSERT [3,4,8,9] to measure and model fires in enclosures yields the following conclusions:

1. A new heat flux gauge (Figs.3 and 4) enables the determination of the distribution of the heat flux on a wall of an enclosure.

2. The previously found $[3,4]$ dependence of the normalized mass loss rate on the normalized opening factor (see Fig. 8a) must be modified to account for low gas temperatures inside the enclosure (see Fig. 8c).

3. Heat fluxes to the wall depend on the local gas temperature (see Figs. 9a, 10a and Figs. 9b, 10b for IMS) if the flame radiation from the fire is not significant (see Figs. 9c, 10c for IMS). This conclusion is further demonstrated by comparing the heat fluxes for Methanol, IMS and toluene in Figs. 12 to 14.

4. Heat flux measurements with Methanol and IMS allow the determination of the convective heat fluxes and the convective heat flux coefficient from the difference of the measured heat flux and the theoretical black body radiation corresponding to the hot gas temperature (see Fig. 15). In addition, using these convective heat fluxes the magnitude of flame radiation can be calculated from the results in Figs. 13a and 14c for toluene together with Eq. 2f. This radiation depends on the size of the fire and the optical thickness of the hot gases.

\section{REFERENCES}

[1] Drysdale, D.D., An Introduction to Fire Dynamics, Wiley, 1990.

[2] Karlsson, B., and Quintiere, J.G, Enclosure Fire Dynamics, CRC Press, 2000.

[3] Delichatsios, M.A, Silcock, G.W.H., Liu, X., Delichatsios, M., and Lee, Y., "Mass Pyrolysis Rates and Excess Pyrolysate in Fully Developed Enclosure Fires," Fire Safety Journal, 39, Iss. 1, pp. 1-21.

[4] Delichatsios, M.A, and Silcock, G.W.H., "Fully Developed Enclosure Fires: Effects of Fuel Type, Fuel Area and Enclosure Geometry," Invited Lecture at the 7th IAFSS Symposium on combustion, pp. 59-73, June 2002.

[5] Tofilo, P., Delichatsios, M.A., Silcock, G.W.H., and Shields, T.J., "Heat Fluxes to the Walls in Enclosure Fires," Proceedings of 6th Asia-Oceania Symposium on Fire Safety and Technology, pp. 108-119, March 2004.

[6] Wickstrom, U., "The Plate Thermometer - A Simple Instrument for Reaching Harmonized Fire Resistance Test," NORDTEST project 609.86, SP Report 1989:03, Swedish National Testing Institute Fire Technology, Boras, 1988.

[7] Ingason, H., and de Ris, J., "Flame Heat Transfer in Storage Geometries,” Dept. of Fire Safety Eng., Rep. LUTVDG/(TVBB-1013), Lund Univ., Sweden, 1996.

[8] Shields, T.J., Silcock, G.W.H., and Flood, F., "Performance of a Single Glazing Assembly Exposed to Enclosure Corner Fires of Increasing Severity," Fire and Materials, 25, pp. 123-152, 2001.

[9] Shields, T.J., Silcock, G., and Flood, F., "Performance of a Single Glazing Assembly Exposed to a Fire in the Centre of an Enclosure," Fire and Materials, 26, pp. 51-75, 2002. 